\title{
HARVEST MANAGEMENT PROBLEM WITH A FRACTIONAL LOGISTIC EQUATION
}

\author{
Melani Barrios ${ }^{1,2, *}$, Gabriela Reyero ${ }^{1}$ and Mabel Tidball ${ }^{3}$ \\ ${ }^{1}$ Departamento de Matemática, Facultad de Ciencias Exactas, Ingeniería y Agrimensura, Universidad Nacional \\ de Rosario, Avda. Pellegrini 250, S2000BTP, Rosario, Argentina \\ ${ }^{2}$ CONICET, Avda. Pellegrini 250, S2000BTP, Rosario, Argentina \\ ${ }^{3}$ CEE-M, Univ. Montpellier, CNRS, INRAE, Institut Agro, Montpellier, France \\ Communicated by Mihály Pituk \\ Original Research Paper \\ Received: Jun 14, 2021 - Accepted: Jul 23, 2021 \\ First published online: October 26, 2021 \\ (c) 2021 The Author(s)

\section{ABSTRACT} \\ In this article, we study a fractional control problem that models the maximization of the profit obtained by exploiting a \\ certain resource whose dynamics are governed by the fractional logistic equation. Due to the singularity of this problem, we \\ develop different resolution techniques, both for the classical case and for the fractional case. We perform several numerical \\ simulations to make a comparison between both cases.
}

\section{KEYWORDS}

fractional logistic equations, Caputo derivative, fractional optimal control problem, natural resource management

\section{MATHEMATICS SUBJECT CLASSIFICATION (2020)}

Primary 26A33; Secondary 34A08, 49-XX, 91B76

\section{INTRODUCTION}

It is very well known that the logistic equation describes the population growth. The continuous Logistic model is described by a first order ordinary differential equation. The model describes the population growth that may be limited by certain factors like population density, [19]. The continuous form of the logistic equation is,

$$
\dot{x}(t)=r x(t)\left(1-\frac{x(t)}{K}\right) .
$$

In the above equation, $x(t)$ indicates population at time $t, r>0$ represents the Malthusian parameter expressing the growth rate of species and $K$ denotes the carrying capacity.

Motivated by its applications in different scientific areas (electricity, magnetism, mechanics, fluid dynamics, medicine, etc., [4, 23, 24, 26]), fractional calculus is in development, which has led to large growth in its study in recent decades. The fractional derivative is a nonlocal operator, [20, 37], making fractional differential equations good candidates for modeling situations in which it is

\footnotetext{
*Corresponding author. E-mail: melani@fceia.unr.edu.ar
} 
important to consider the history of the phenomenon studied [22], unlike the models with a classical derivative where this is not taken into account. There are several definitions of fractional derivatives. The most commonly used are the Riemann-Liouville fractional derivative and the Caputo fractional derivative. It is important to note that while the Riemann-Liouville fractional derivative [36], is historically the most studied approach to fractional calculus, the Caputo fractional derivative is more popular among physicists and scientists due to the fact that the formulation of initial value problems with this type of derivative is more similar to the formulation with the classical derivative.

The fractional order logistic equation has been discussed in the literature, [15, 34]. A detailed study of existence, uniqueness, stability and approximate solutions of this equation can be found in $[1,7,13,21,27,40]$.

When the logistic equation is used to describe the natural evolution of a species, it is logical to think about the exploitation of this resource. For this reason consider a natural resource management problem using fractional derivatives. The fractional equation can better represent the evolution of the resource, in fact in this work we want to see how a fractional derivative affects the optimal extraction decision.

The principal objective of this work is to solve an optimal control problem of extraction management. Due to the nature of the problem, it can be turned into a Fractional Variational problem. In the last years numerous works have been developed extending the theory of the variational calculus [41] to problems of fractional variational calculus $[2,12,14,25,32,33,38]$. With the purpose of solving fractional variational problems, there exist two theorems of optimality conditions: an Euler-Lagrange equation which involves Caputo and Riemann-Liouville fractional derivatives [3, 5, 6, 10, 30, 31], and another Euler-Lagrange equation that involves only Caputo derivatives [8, 11, 16, 28]. As it is not yet known which of these theorems is the most suitable for solving this type of problem, for qualitative reasons the second method will be used to solve our problem. The structure of this article is as follows: in Section 2, the classical control problem and its solution, the fundamental concept of fractional derivatives and fractional control and variational problems, are presented. In Section 3, the solution of a fractional control problem, the comparison between both problems and numerical approximations of the solutions are discussed. Finally, the last section, is dedicated to the conclusions.

\section{PRELIMINARIES}

Some known facts are summarised for the reader's convenience.

\subsection{A simple optimal control problem}

We present a classical optimal control model, which consists of maximizing the total extraction (or harvest) of a certain renewable resource in the interval $[0, T]$, where a first order ordinary differential equation describes the evolution of the dynamics,

$$
\left\{\begin{array}{l}
\max \int_{0}^{T} e^{-\delta t} h(x(t)) \mathrm{d} t \\
\dot{x}(t)=r x(t)\left(1-\frac{x(t)}{K}\right)-h(x(t)) \\
x(0)=x_{0} \\
x(T)=x_{T} \\
h_{\min } \leq h(x(t)) \leq h_{\max } .
\end{array}\right.
$$

$T$ represents the final time, $x_{0}$ the initial condition, $x_{T}$ the final condition, $h_{\min }$ and $h_{\max }$ minimum and maximum harvest and $e^{-\delta t}$ represents a discount factor with $\delta \geq 0$ the instantaneous annual discount rate which can be zero.

The natural resource follows a logistic growth function, where $r>0$ is the intrinsic growth rate, $K>0$ the carrying capacity of the resource and $h(t)$ is the harvest.

Now we will proceed to solve the problem (2.1). 
Clearing $h(x(t))$ from the dynamics and replacing it in the functional to maximize results,

$$
\left\{\begin{array}{l}
\max \int_{0}^{T} e^{-\delta t}\left[r x(t)\left(1-\frac{x(t)}{K}\right)-\dot{x}(t)\right] \mathrm{d} t \\
x(0)=x_{0} \\
x(T)=x_{T} \\
h_{\min } \leq h(x(t)) \leq h_{\max },
\end{array}\right.
$$

which is a variational problem with Lagrangian

$$
L(t, x, \dot{x})=e^{-\delta t}\left[r x(t)\left(1-\frac{x(t)}{K}\right)-\dot{x}(t)\right] .
$$

Then, its Euler-Lagrange equation results

$$
\frac{\partial L}{\partial x}-\frac{d}{d t} \frac{\partial L}{\partial \dot{x}}=0,
$$

from which is obtained

$$
r\left(1-\frac{2}{K} x(t)\right)-\delta=0 .
$$

That is, the optimal population is constant

$$
x^{*}=\frac{K}{2}\left(1-\frac{\delta}{r}\right)
$$

while the optimal harvest is

$$
h^{*}=r x^{*}\left(1-\frac{x^{*}}{K}\right)
$$

then,

$$
h^{*}=\frac{K}{4} \frac{r^{2}-\delta^{2}}{r} .
$$

REMARK 2.1. It can be seen that since the solution of the Euler-Lagrange equation $x^{*}(t)$, is a constant function, it will not be able to verify the imposed boundary conditions. For this reason, it can be said that the problem is singular.

For its resolution, the Nearest Feasible Paths theorem will be used, and its proof can be seen in [39]. This theorem, as its name implies, shows that the optimal solution is to go as fast as possible to $x^{*}(t)$. With this result, the method consists in finding $x_{a}(t)$ the solution to the problem that begins in $x(0)=x_{0}$ and uses the minimum harvest $h_{\min }$ if $x_{0}<x^{*}(t)$ or uses the maximum harvest $h_{\max }$ if $x_{0}>x^{*}(t)$, until it reaches the value $x^{*}(t)$ in the time $t_{\min }$ to be determined.

Then it must be found $x_{b}(t)$, a solution to the problem that uses the minimum harvest $h_{\min }$ if $x_{T}>x^{*}(t)$ or uses the maximum harvest $h_{\max }$ if $x_{T}<x^{*}(t)$, until it reaches the value $x(T)=x_{T}$, from the time $t_{\max }$ to be determined so that it is the first time when $x_{b}(t)$ gets the value $x^{*}(t)$.

In summary, the following optimal solution is obtained

$$
x^{*}(t)=\left\{\begin{array}{lll}
x_{a}(t) & \text { if } & 0 \leq t \leq t_{\min } \\
\frac{K}{2}\left(1-\frac{\delta}{r}\right) & \text { if } & t_{\min } \leq t \leq t_{\max } \\
x_{b}(t) & \text { if } & t_{\max } \leq t \leq T
\end{array}\right.
$$

and the optimal harvest obtained with this procedure is

$$
h^{*}(t)=\left\{\begin{array}{lll}
h_{\min } & \text { if } & x(t)>x^{*}(t) \\
\frac{K}{4} \frac{r^{2}-\delta^{2}}{r} & \text { if } & x(t)=x^{*}(t) \\
h_{\max } & \text { if } & x(t)<x^{*}(t),
\end{array}\right.
$$

taking into account that for it must be satisfied $h_{\min } \leq \frac{K}{4} \frac{r^{2}-\delta^{2}}{r} \leq h_{\max }$. 
REMARK 2.2. The problem without discount is obtained as a particular case, writing $\delta=0$ in the equations (2.5) and (2.6).

In the next section, this problem will be solved but in its fractional version. To see more about fractional calculus refer to appendix at the end of the paper.

\subsection{Fractional control and variational problems}

To solve a fractional control problem, tools of fractional variational analysis will be used. For this, a brief introduction to them is presented.

Consider the following problem of the fractional calculus of variations: find a function $x \in{ }_{a}^{\alpha} E$ that optimizes (minimizes or maximizes) the functional

$$
J(x)=\int_{a}^{b} L\left(t, x,{ }_{a}^{C} D_{t}^{\alpha} x\right) \mathrm{d} t,
$$

with a Lagrangian $L \in C^{1}\left([a, b] \times \mathbb{R}^{2}\right)$ and

$$
{ }_{a}^{\alpha} E=\left\{x:[a, b] \rightarrow \mathbb{R}: x \in C^{1}([a, b]),{ }_{a}^{C} D_{t}^{\alpha} x \in C([a, b])\right\},
$$

subject to the boundary conditions $x(a)=x_{a}, x(b)=x_{b}$.

Now the Euler-Lagrange equation for this problem will be stated, its proof is in [28].

THEOREM 2.3. Let $x$ be an optimizer of $J$ in ${ }_{a}^{\alpha} E$ with $L \in C^{2}\left([a, b] \times \mathbb{R}^{2}\right)$ subject to boundary conditions $x(a)=x_{a}, x(b)=x_{b}$, then $x$ satisfies the fractional Euler-Lagrange differential equation

$$
\frac{\partial L}{\partial x}+{ }_{t}^{C} D_{b}^{\alpha}\left[\frac{\partial L}{\partial{ }_{a}^{C} D_{t}^{\alpha} x}\right]=0 .
$$

\section{MAIN RESULT}

Now we are ready to prove the following main result.

\subsection{Fractional model}

The fractional version of the problem proposed in the first section is,

$$
\left\{\begin{array}{l}
\max \int_{0}^{T} e^{-\delta t} h(x(t)) \mathrm{d} t \\
{ }_{0}^{C} D_{t}^{\alpha}[x](t)=r x(t)\left(1-\frac{x(t)}{K}\right)-h(x(t)) \\
x(0)=x_{0} \\
x(T)=x_{T} \\
h_{\text {min }} \leq h(x(t)) \leq h_{\text {max }} .
\end{array}\right.
$$

The only difference with the problem exposed in Section 2.1, is that in the dynamic equation

$$
{ }_{0}^{C} D_{t}^{\alpha}[x](t)=r x(t)\left(1-\frac{x(t)}{K}\right)-h(x(t)),
$$

the first-order derivative no longer appears, but now intervenes ${ }_{0}^{C} D_{t}^{\alpha}[x](t)$, the left Caputo fractional derivative of order $0<\alpha \leq 1$.

The derivatives with fractional order contains partially or totally the history, temporary future or the spatial behavior of the function, averaged in some way. This transforms the fractional differential equations on suitable candidates for the modeling of memory phenomenon or subsequent effects, those in which what happens at a point on the space or at an instant of time depends on an interval (spatial or temporal) that contains the point or the instant.

The Riemann-Liouville fractional derivative had an important role in the development of the fractional calculus theory, and was used successfully in strictly mathematical applications. But when it was using for mathematical modeling of real physical phenomena with fractional differential

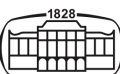


equations, the problem of the initial conditions also of fractional order emerged. These types of conditions are not physically interpretable and present a considerable obstacle when making practical use of fractional calculus. The Caputo differential operator, in contrast to the RiemannLiouville operator, uses derivatives of integer order as initial conditions, that is, initial values that are physically interpretable as in the models with integer derivatives. The definition that follows represented a notable practical advance in the study of physical phenomena such as those of the viscoelastic type and others.

Finally, the fractional derivative at $t$ of a function $x$ is a non-local operator, depending on past values of $x$ (left derivatives) or future values of $x$ (right derivatives). In physics, the right fractional derivative of $x(t)$ is interpreted as a future state of the process $x(t)$. For this reason, the right derivative is usually neglected in applications, when the present state of the process does not depend on the results of the future development. However, the left fractional derivative of $x(t)$ is interpreted as a past state of the process $x(t)$, in which memory effects intervene.

Since the evolution of a certain resource depends on its past, we have decided to choose the left Caputo fractional derivative for modeling its evolution, see [17].

Now the problem (3.1) will be solved.

Clearing $h(x(t))$ from the dynamic equation (3.2) and replacing it in the functional to maximize, it results,

$$
\left\{\begin{array}{l}
\max \int_{0}^{T} e^{-\delta t}\left[r x(t)\left(1-\frac{x(t)}{K}\right)-{ }_{0}^{C} D_{t}^{\alpha}[x](t)\right] \mathrm{d} t \\
x(0)=x_{0} \\
x(T)=x_{T} \\
h_{\min } \leq h(x(t)) \leq h_{\text {max }} .
\end{array}\right.
$$

This is a variational problem, now fractional, with fractional Lagrangian

$$
L\left(t, x,{ }_{0}^{C} D_{t}^{\alpha}[x]\right)=e^{-\delta t}\left[r x(t)\left(1-\frac{x(t)}{K}\right)-{ }_{0}^{C} D_{t}^{\alpha}[x](t)\right],
$$

belonging to $C^{2}\left([0, T] \times \mathbb{R}^{2}\right)$.

Using Theorem 2.3, its fractional Euler-Lagrange equation (2.7) results,

$$
\frac{\partial L}{\partial x}+{ }_{t}^{C} D_{T}^{\alpha}\left[\frac{\partial L}{\partial_{0}^{C} D_{t}^{\alpha}[x]}\right]=0
$$

from which we have

$$
e^{-\delta t} r\left(1-\frac{2}{K} x(t)\right)-\delta(T-t)^{1-\alpha} e^{-\delta T} E_{1,2-\alpha}(\delta(T-t))=0
$$

where $E_{1,2-\alpha}(\delta(T-t))$ is the Mittag-Leffler function of two parameters, defined in equation (3.11).

We can conclude that the optimal solution is

$$
x_{\alpha}^{*}(t)=\frac{K}{2}\left(1-\frac{\delta}{r}(T-t)^{1-\alpha} e^{-\delta(T-t)} E_{1,2-\alpha}(\delta(T-t))\right) .
$$

And the optimal harvest is obtained from this expression

$$
h_{\alpha}^{*}(t)=r x_{\alpha}^{*}(t)\left(1-\frac{x_{\alpha}^{*}(t)}{K}\right)-{ }_{0}^{C} D_{t}^{\alpha}\left[x_{\alpha}^{*}\right](t) .
$$

Note that in the classical case the optimal population is constant, which is not happening in this case, therefore in the optimal harvest a term appears with the Caputo derivative of $x_{\alpha}^{*}(t)$, that due to its difficulty, we must calculate it numerically.

REMARK 3.1. We can see that, as in the classical case, the optimal solution $x_{\alpha}^{*}(t)$, although it is not a constant, will also not verify the established boundary conditions. This means that we are once again faced with a singular problem. 
For its resolution, it would be possible to resort again to a Nearest Feasible Paths theorem, but in a fractional version. However, it has not been carried out yet because the proof of the theorem requires the use of a fractional Green theorem, which at the moment is only available for rectangular regions, [35], and its version is necessary for all types of regions.

REMARK 3.2. The solution of the problem without discount $(\delta=0)$ is a particular case of the solution of the problem (3.1).

The optimal population is $x_{\alpha}^{*}(t)=\frac{K}{2}$ and the optimal harvest is obtained from this expression $h_{\alpha}^{*}(t)=\frac{r K}{4}$.

It can be observed that the solution is the same for the classical case. In the case with $\delta=0$, the solution and the optimal harvest is the same for all $0<\alpha \leq 1$. The reason of this is that there is no time-dependent factor that multiplies ${ }_{0}^{C} D_{t}^{\alpha}\left[x_{\alpha}^{*}\right](t)$ in the fractional variational problem resulting from taking $\delta=0$.

Its fractional Euler-Lagrange equation is

$$
r\left(1-\frac{2}{K} x(t)\right)+{ }_{t}^{C} D_{T}^{\alpha}[(-1)]=0,
$$

then,

$$
r\left(1-\frac{2}{K} x(t)\right)=0 .
$$

Since the Caputo derivative of a constant is also zero, as in the case of the classical derivative, then the same Euler-Lagrange equation is obtained for all $0<\alpha \leq 1$ and its solution is independent of that value.

To perform a graphic analysis of the solutions to the discounted problem in both the fractional and classical cases, we must see a specific example.

\subsection{Example and comparison}

Consider $r, K, \delta, x_{0}, x_{T}, T, h_{\min }$ and $h_{\max }$ as in the example The Pacific Halibut Fishery, in [18], and $0<\alpha \leq 1$.

The problem results,

$$
\left\{\begin{array}{l}
\max \int_{0}^{10} e^{-0.01 t} h(x(t)) \mathrm{d} t \\
{ }_{0}^{C} D_{t}^{\alpha}[x](t)=0.71 x(t)\left(1-\frac{x(t)}{80.5}\right)-h(x(t)) \\
x(0)=x_{0} \\
x(10)=x_{10} \\
10 \leq h(x(t)) \leq 15 .
\end{array}\right.
$$

Solving this as in the previous section, from (3.3) and (3.4), one obtains

$$
x_{\alpha}^{*}(t)=40.25\left(1-\frac{0.01}{0.71}(10-t)^{1-\alpha} e^{-0.01(10-t)} E_{1,2-\alpha}(0.01(10-t))\right) .
$$

While the optimal harvest is

$$
h_{\alpha}^{*}(t)=0.71 x_{\alpha}^{*}(t)\left(1-\frac{x_{\alpha}^{*}(t)}{80.5}\right)-{ }_{0}^{C} D_{t}^{\alpha}\left[x_{\alpha}^{*}\right](t) .
$$

In Figure 1, the solutions corresponding to $\alpha=1, \alpha=0.9, \alpha=0.8, \alpha=0.6$ and $\alpha=0.4$ were shown. It can be noticed that when $\alpha$ tends to 1 , the solutions converge to the classical solution. Also, it can be observed that the solutions not verify the established boundary conditions, their initial and final values change.

From now on, a fixed value of $\alpha$ is assumed to evade the problem of needing a fractional Nearest Feasible Paths theorem, being able to obtain the boundary conditions $x(0)$ and $x(10)$ of this fractional solution, in order to make a comparison with the classical problem (with $\alpha=1$ ). 


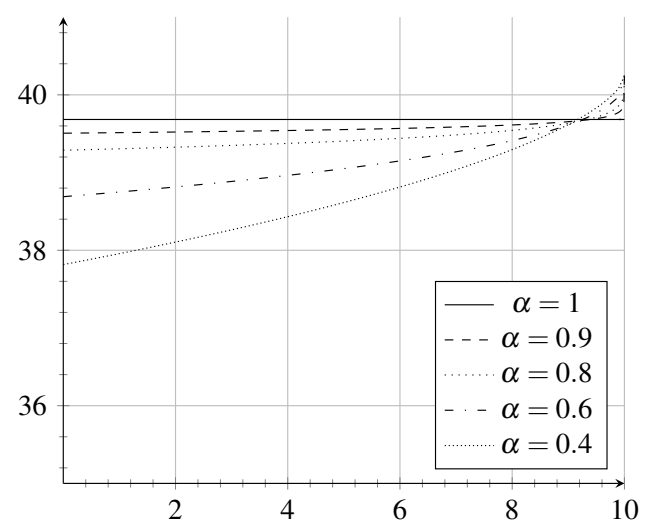

FIgURE 1. Solutions for $\alpha=1, \alpha=0.9, \alpha=0.8, \alpha=0.6$ and $\alpha=0.4$.

The following problem will be considered,

$$
\left\{\begin{array}{l}
\max \int_{0}^{10} e^{-0.01 t} h(x(t)) \mathrm{d} t \\
{ }_{0}^{C} D_{t}^{\alpha}[x](t)=0.71 x(t)\left(1-\frac{x(t)}{80.5}\right)-h(x(t)) \\
x(0)=38.6896 \\
x(10)=40.25 \\
10 \leq h(x(t)) \leq 15 .
\end{array}\right.
$$

A comparison of the results will be made between $\alpha=1$ (classical version) and $\alpha=0.6$ (fractional version).

Solving the problem in its classical version as in the previous section, using (2.2) and (2.4) we have $x^{*}(t)=39.6831$ and $h^{*}(t)=14.2859$.

It can be observed that $x^{*}(t)$ does not verify the required boundary conditions, therefore, the Nearest Feasible Paths theorem will be used to solve the problem.

As explained in the last section we obtain the optimal solution as a most rapid approach to $x$ *,

$$
x^{*}(t)=\left\{\begin{array}{lll}
\frac{20.9703+62.3013 e^{0.388979 t}}{1.1523+e^{0.388979 t}} & \text { if } & 0 \leq t \leq 0.2322 \\
39.6831 & \text { if } & 0.2322 \leq t \leq 9.8678 \\
\frac{889.931+62.3013 e^{0.388979 t}}{48.9008+e^{0.388979 t}} & \text { if } & 9.8678 \leq t \leq 10=T
\end{array}\right.
$$

and the optimal harvest obtained with this procedure is

$$
h^{*}(t)=\left\{\begin{array}{lll}
10 & \text { if } & 0 \leq t \leq 0.232235 \\
14.2859 & \text { if } & 0.232235 \leq t \leq 9.8678 \\
10 & \text { if } & 9.8678 \leq t \leq 10=T .
\end{array}\right.
$$

The solution of its fractional version as in equation (3.5) is

$$
x_{0.6}^{*}(t)=40.25\left(1-\frac{0.01}{0.71}(10-t)^{0.4} e^{-0.01(10-t)} E_{1,1.4}(0.01(10-t))\right) .
$$

While the optimal harvest as in equation (3.6) is

$$
h_{0.6}^{*}(t)=0.71 x_{0.6}^{*}(t)\left(1-\frac{x_{0.6}^{*}(t)}{80.5}\right)-{ }_{0}^{C} D_{t}^{0.6}\left[x_{0.6}^{*}\right](t) .
$$




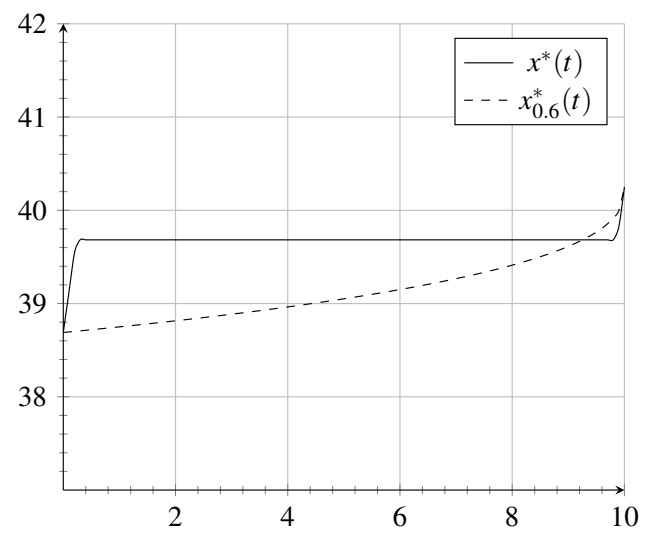

FiguRE 2. Optimal populations of classic and fractional problems.

In Figure 2, the optimal populations corresponding to both cases can be observed. It can be noticed that the optimal solution given by the fractional problem, $x_{0.6}^{*}(t)$, is lower than the optimal population of the classical problem, $x^{*}(t)$, most of the time. It means that the version with $\alpha=0.6$ shows a deterioration of the state of the stock with respect to the case $\alpha=1$ which is only recovered at the end by the fact that it has to verify the final condition.

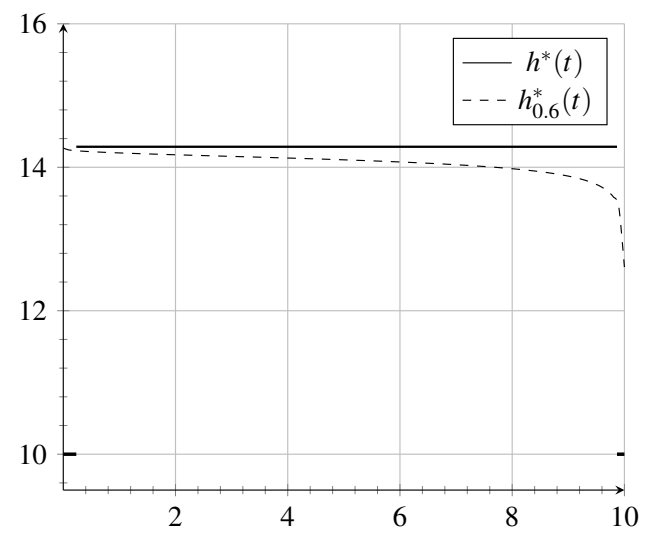

FIGURE 3. Optimal harvests of classical and fractional problems.

In Figure 3, the optimal harvests are considered. Since ${ }_{0}^{C} D_{t}^{0.6}\left[x_{0.6}^{*}\right](t)$ cannot be obtained exactly, we will proceed to use a fractional numerical method of L1 type, [9, 29]. It can be noticed that although with the fractional problem the extraction of the resource is smaller than with the classical case for most of the points, which is logical because in the fractional model the resource grows more slowly, it only decreases to a greater extent at the end of the interval near $T$ and until the final extraction in $T$ turns out being larger than in the classical case.

This way, it is possible to make a comparison of the profit obtained in each case,

Classical case profit: 134.411.

Fractional case profit: 133.828

To make an analysis of this, consider the following graphic of the resources evolution without harvest with the given initial condition.

It can be noted that the use of a fractional dynamic equation, which makes the resource grow more slowly as in Figure 4, does not vary considerably the profit compared to the classical case. 


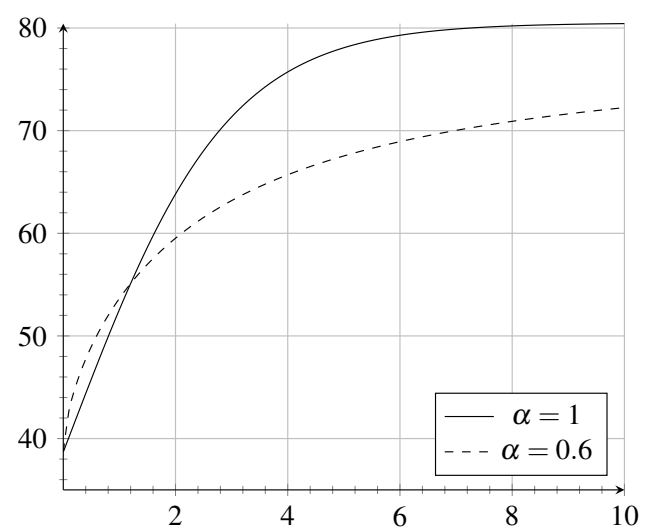

Figure 4. Populations of the classical and fractional problems without harvest.

Lastly, it can be stated what happens to the population $x(t)$ if we take the optimal harvest of the classic problem $h_{1}^{*}$ and consider it in the fractional dynamic equation of the resource.

We must solve

$$
\left\{\begin{array}{l}
{ }_{0}^{C} D_{t}^{\alpha}[x](t)=0.71 x(t)\left(1-\frac{x(t)}{80.5}\right)-14.2859 \\
x(0)=38.6896 .
\end{array}\right.
$$

Since this equation has no known exact solution, it will be approximated using the Adams fractional method, which consists of using Euler's method to obtain $u_{n+1}^{P}$ (predictor), and the trapezoidal fraction rule to get $u_{n+1}$ (corrector),

$$
\left\{\begin{array}{l}
u_{n+1}^{P}=\sum_{j=0}^{m-1} \frac{t_{n+1}^{j}}{j !} u_{0}^{j}+\sum_{j=0}^{n} b_{j, n+1} f\left(t_{j}, u_{j}\right), \\
u_{n+1}=\sum_{j=0}^{m-1} \frac{t_{n+1}^{j}}{j !} u_{0}^{j}+\sum_{j=0}^{n} a_{j, n+1} f\left(t_{j}, u_{j}\right)+a_{n+1, n+1} f\left(t_{n+1}, u_{n+1}^{P}\right) .
\end{array}\right.
$$

For more details refer to [9, 29].

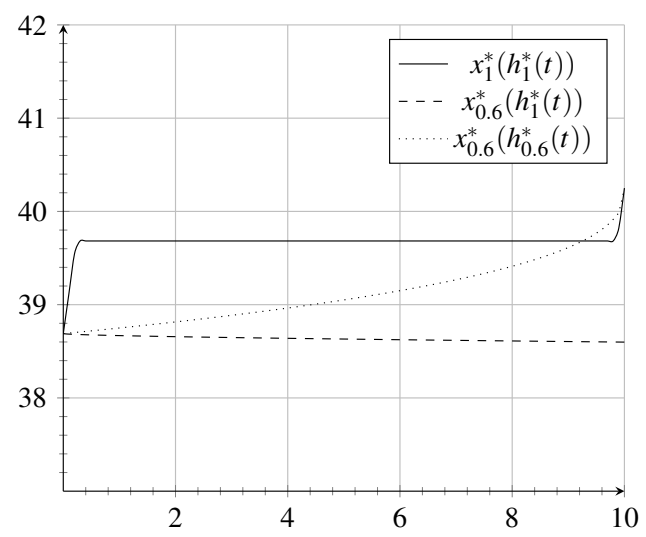

Figure 5. Optimal populations of the classical and fractional with different harvest problems.

In Figure 5, the population obtained from the fractional dynamic equation, taking the optimal harvest of the classical control problem, is lower than the obtained by taking the optimal harvest of the fractional problem, as we expected. 
Furthermore, if we assume that the "true evolution" of the resource is considering $\alpha=0.6$ in the dynamic equation and that the harvesting agency considers the dynamic equation of $\alpha=1$ to be erroneous, the loss is only due to the difference between harvest. Also note that the difference between the profit obtained could be more significant if the instantaneous profit function, which in this case is only the harvest, was also depending on the stock as in Remark 1.

\section{CONCLUSIONS}

In this article, we have studied a fractional control problem that models the maximization of the profit obtained by exploiting a certain resource. An explanation of the proposed model has been made. Due to the singularity of the problem, different resolution techniques have been developed: for the classical case an Euler-Lagrange equation and the Nearest Feasible Paths theorem; and for the fractional case a fractional Euler-Lagrange equation and numerical methods. Although we have seen the need of a non-existent fractional Nearest Feasible Paths theorem, we have been able to make a comparison between the classical and fractional results for a certain value of the fractional order. It is also observed that the order of time fractional derivative significantly affects the population growth. Hence, we conclude that fractional derivatives may be more suitable for modeling the evolution of natural resources that naturally have a resilience problem. As a future investigation, it is proposed that the extension of the fractional Nearest Feasible Paths theorem should be explored and the optimal control problem should be extended for more complex instantaneous profit functions.

\section{ACKNOWLEDGEMENTS}

This work was partially supported by Universidad Nacional de Rosario a través del proyecto ING568 "Problemas de Control Óptimo Fraccionario" 80020190100005UR "Ecuaciones diferenciales fraccionarias en modelos de evolución poblacional”. The first author was also supported by CONICET through a PhD fellowship.

\section{REFERENCES}

[1] Abbas, S., Banerjee, M., And Momani, S. Dynamical analysis of fractional-order modified logistic model. Computers \& Mathematics with Applications 62, 3 (2011), 1098-1104.

[2] Agrawal, O. P. Formulation of Euler-Lagrange equations for fractional variational problems. Journal of Mathematical Analysis and Applications 272, 1 (2002), 368-379.

[3] Agrawal, O. P. Generalized Euler-Lagrange equations and transversality conditions for FVPs in terms of the Caputo derivative. Fournal of Vibration and Control 13, 9-10 (2007), 1217-1237.

[4] Almeida, R., Malinowska, A. B., And Torres, D. F. A fractional calculus of variations for multiple integrals with application to vibrating string. Fournal of Mathematical Physics 51, 3 (2010), 033503.

[5] Almeida, R., Malinowska, A. B., And Torres, D. F. Fractional Euler-Lagrange differential equations via Caputo derivatives. In Fractional dynamics and control. Springer, 2012, pp. 109118.

[6] Almeida, R., AND Torres, D. F. Necessary and sufficient conditions for the fractional calculus of variations with Caputo derivatives. Communications in Nonlinear Science and Numerical Simulation 16, 3 (2011), 1490-1500.

[7] Amarti, Z., Nurkholipah, N., Anggriani, N., And Supriatna, A. Numerical solution of a logistic growth model for a population with Allee effect considering fuzzy initial values and fuzzy parameters. In IOP (Institute of Physics) Conference Series: Materials Science and Engineering (2018), vol. 332, p. 1.

[8] Antunes, P. R., And Ferreira, R. A. Analysis of a class of boundary value problems depending on left and right Caputo fractional derivatives. Communications in Nonlinear Science and Numerical Simulation 48 (2017), 398-413. 
[9] Baleanu, D., Diethelm, K., Scalas, E., And Trujillo, J. J. Fractional calculus: models and numerical methods. World Scientific, 2012.

[10] Baleanu, D., ANd Trujillo, J. J. On exact solutions of a class of fractional Euler-Lagrange equations. Nonlinear Dynamics 52, 4 (2008), 331-335.

[11] Barrios, M., And Reyero, G. An Euler-Lagrange Equation only Depending on Derivatives of Caputo for Fractional Variational Problems with Classical Derivatives. Statistics, Optimization \& Information Computing 8(2), 10.19139/soic-2310-5070-865 (2020), 590-601.

[12] Barrios, M., Reyero, G., And Lombardi, A. Método numérico de tipo L1 para problemas variacionales fraccionarios. In Proceedings of the Congreso de Mecánica Computacional (2018), vol. XXXVI, pp. 1089-1098.

[13] Barrios, M., Reyero, G., ANd Tidball, M. Estabilidad de una generalización de ecuaciones logísticas fraccionarias. In Proceedings of the VII Congreso de Matemática Aplicada, Computacional e Industrial (2019).

[14] Bastos, N. R. Calculus of variations involving Caputo-Fabrizio fractional differentiation. Statistics, Optimization \& Information Computing 6, 1 (2018), 12-21.

[15] Bhalekar, S., And Daftardar-GejJi, V. Solving fractional-order logistic equation using a new iterative method. International fournal of Differential Equations 2012 (2012).

[16] BlaszczyK, T., AND CiesielsKi, M. Numerical solution of Euler-Lagrange equation with Caputo derivatives. Advances in Applied Mathematics and Mechanics 9, 1 (2017), 173-185.

[17] Caputo, M. C., ANd Torres, D. F. Duality for the left and right fractional derivatives. Signal Processing 107 (2015), 265-271.

[18] CLARK, C. Mathematical bioeconomics: the optimal management of renewable resources. John Wiley and Sons Inc.: New York, 1990.

[19] Cushing, J. M. An introduction to structured population dynamics. SIAM, 1998.

[20] Diethelm, K. The analysis of fractional differential equations: An application-oriented exposition using differential operators of Caputo type. Springer Science \& Business Media, 2010.

[21] El-SaYed, A., EL-Mesiry, A., AND EL-SAKA, H. On the fractional-order logistic equation. Applied Mathematics Letters 20, 7 (2007), 817-823.

[22] Ferrari, A., And Marcus, E. S. Study of a fractional-order model for HIV infection of CD4+ T-cells with treatment. Fournal of Fractional Calculus and Applications 11, 2 (2020), 12-22.

[23] Goos, D., Reyero, G., Roscani, S., and Santillan Marcus, E. On the initial-boundary-value problem for the time-fractional diffusion equation on the real positive semiaxis. International Journal of Differential Equations 2015 (2015).

[24] Hilfer, R., ET AL. Applications of fractional calculus in physics, vol. 35. World Scientific Singapore, 2000.

[25] JAHANSHAHI, S., AND ToRRES, D. F. A simple accurate method for solving fractional variational and optimal control problems. fournal of Optimization Theory and Applications 174, 1 (2017), 156-175.

[26] Kilbas, A. A., Srivastava, H. M., And Trujillo, J. J. Theory and applications of fractional differential equations, vol. 204. Elsevier, 2006.

[27] Kumar, D., Singh, J., Al Qurashi, M., And Baleanu, D. Analysis of logistic equation pertaining to a new fractional derivative with non-singular kernel. Advances in Mechanical Engineering 9, 2 (2017), 1687814017690069.

[28] Lazo, M. J., AND Torres, D. F. The DuBois-Reymond fundamental lemma of the fractional calculus of variations and an Euler-Lagrange equation involving only derivatives of Caputo. fournal of Optimization Theory and Applications 156, 1 (2013), 56-67.

[29] LI, C., AND ZENG, F. Numerical methods for fractional calculus, vol. 24. CRC Press, 2015.

[30] Malinowska, A. B., Odzijewicz, T., And Torres, D. F. Advanced methods in the fractional calculus of variations. Springer, 2015.

[31] Malinowska, A. B., AND Torres, D. F. Introduction to the fractional calculus of variations. World Scientific Publishing Company, 2012. 
[32] Malmir, I. A general framework for optimal control of fractional nonlinear delay systems by wavelets. Statistics, Optimization \& Information Computing 8, 4 (2020), 858-875.

[33] Mohammadi, F., Moradi, L., And Conte, D. Discrete chebyshev polynomials for solving fractional variational problems. Statistics, Optimization \& Information Computing (2021).

[34] Momani, S., ANd QARAlleh, R. Numerical approximations and Padé approximants for a fractional population growth model. Applied Mathematical Modelling 31, 9 (2007), 1907-1914.

[35] Odzijewicz, T., Malinowska, A. B., ANd Torres, D. F. Fractional variational calculus with classical and combined Caputo derivatives. Nonlinear Analysis: Theory, Methods \& Applications 75, 3 (2012), 1507-1515.

[36] Oldham, K., AND SPANIER, J. The fractional calculus theory and applications of differentiation and integration to arbitrary order. Elsevier, 1974.

[37] Podlubny, I. Fractional differential equations: an introduction to fractional derivatives, fractional differential equations, to methods of their solution and some of their applications. Elsevier, 1998.

[38] Rangaig, N. A. New aspects on the fractional euler-lagrange equation with non-singular kernels. Journal of Applied Mathematics and Computational Mechanics 19, 4 (2020), 89-100.

[39] Sethi, S. P. Nearest feasible paths in optimal control problems: Theory, examples, and counterexamples. Journal of Optimization Theory and Applications 23, 4 (1977), 563-579.

[40] Sweilam, N., Khader, M., AND MAhdy, A. Numerical studies for solving fractional-order Logistic equation. International fournal of Pure and Applied Mathematics 78, 8 (2012), 1199-1210.

[41] van Brunt, B. The Calculus of Variations. Springer, 2004.

\section{APPENDIX: INTRODUCTION TO FRACTIONAL CALCULUS}

In this section certain definitions and properties of the fractional calculus will be presented. For more details refer to [20, 36, 37].

DEFINITION 3.3. The Mittag Leffler function with parameters $\alpha, \beta$, is defined by

$$
E_{\alpha, \beta}(z)=\sum_{k=0}^{\infty} \frac{z^{k}}{\Gamma(\alpha k+\beta)},
$$

for all $z \in \mathbb{C}$.

DEFINITION 3.4. The Riemann-Liouville fractional integral operator of order $\alpha \in \mathbb{R}_{0}^{+}$is defined in $L^{1}[a, b]$ by

$$
{ }_{a} I_{t}^{\alpha}[f](t)=\frac{1}{\Gamma(\alpha)} \int_{a}^{t}(t-s)^{\alpha-1} f(s) \mathrm{d} s .
$$

DEFINITION 3.5. (Left and Right Caputo Fractional Derivatives)

The left and right Caputo fractional derivatives of order $\alpha \in \mathbb{R}_{0}^{+}$are defined, respectively, by

and

$$
{ }_{a}^{C} D_{t}^{\alpha}[f](t)=\frac{1}{\Gamma(n-\alpha)} \int_{a}^{t}(t-s)^{n-1-\alpha} \frac{d^{n}}{d s^{n}} f(s) \mathrm{d} s
$$

$$
{ }_{t}^{C} D_{b}^{\alpha}[f](t)=\frac{(-1)^{n}}{\Gamma(n-\alpha)} \int_{t}^{b}(s-t)^{n-1-\alpha} \frac{d^{n}}{d s^{n}} f(s) \mathrm{d} s,
$$

with $n=\lceil\alpha\rceil$ and $\frac{d^{n} f}{d t^{n}} \in L^{1}[a, b]$.

Open Access statement. This is an open-access article distributed under the terms of the Creative Commons AttributionNonCommercial 4.0 International License (https://creativecommons.org/licenses/by-nc/4.0/), which permits unrestricted use, distribution, and reproduction in any medium for non-commercial purposes, provided the original author and source are credited, a link to the CC License is provided, and changes - if any - are indicated. 